\title{
The effect of oral diabetes medications on glycated haemoglobin (HbA1c) in Asians in primary care: a retrospective cohort real-world data study
}

Hao Sen Andrew Fang ${ }^{1 *}$ (D), Qiao Gao ${ }^{2}$, Wei Ying Tan², Mong Li Lee ${ }^{2,3}$, Wynne Hsu²,3 and Ngiap Chuan Tan 1,4

\begin{abstract}
Background: Clinical trials have demonstrated that initiating oral anti-diabetic drugs (OADs) significantly reduce glycated hemoglobin (HbA1c) levels. However, variability in lifestyle modifications and OAD adherence impact on their actual effect on glycemic control. Furthermore, evidence on dose adjustments and discontinuation of OAD on $\mathrm{HbA} 1 \mathrm{c}$ is lacking. This study aims to use real-world data to determine the effect of OAD initiation, up-titration, down-titration, and discontinuation on HbA1c levels, among Asian patients managed in primary care.

Methods: A retrospective cohort study over a 5-year period, from Jan 2015 to Dec 2019 was conducted on a cohort of multi-ethnic adult Asian patients with clinical diagnosis of type 2 diabetes mellitus (T2DM) managed by a network of primary care clinics in Singapore. Nine OADs from five different classes (biguanides, sulphonyurea, dipeptidyl peptidase-4 [DPP-4] inhibitors, sodium-glucose cotransporter-2 [SGLT-2] inhibitors, and alpha-glucosidase inhibitors) were evaluated. Patients were grouped into "No OAD", "Non-titrators," and "Titrators" cohorts based on prescribing patterns. For the "Titrators" cohort, the various OAD titrations were identified. Subsequently, a descriptive analysis of $\mathrm{HbA1c}$ values before and after each titration was performed to compute a mean difference for each unique titration identified.

Results: Among the cohort of 57,910 patients, 43,338 of them had at least one OAD titration, with a total of 76,990 pairs of $\mathrm{HbA} 1 \mathrm{c}$ values associated with an OAD titration. There were a total of 206 unique OAD titrations. Overall, initiation of OADs resulted in a reduction of $\mathrm{HbA1c}$ by 3 to $12 \mathrm{mmol} / \mathrm{mol}(0.3$ to $1.1 \%)$, respectively. These results were slightly lower than those reported in clinical trials of 6 to $14 \mathrm{mmol} / \mathrm{mol}(0.5$ to $1.25 \%)$. The change of HbA1c levels due to up-titration, down-titration, and discontinuation were -1 to $-8 \mathrm{mmol} / \mathrm{mol}(-0.1$ to $-0.7 \%),+1$ to 7 $\mathrm{mmol} / \mathrm{mol}(+0.1$ to $+0.6 \%)$, and +2 to $11 \mathrm{mmol} / \mathrm{mol}(+0.2$ to $+1.0 \%)$, respectively. The HbA1c lowering effect of initiating newer OADs, namely DPP-4 inhibitors and SGLT-2 inhibitors was 8 to $11 \mathrm{mmol} / \mathrm{mol}(0.7$ to $0.9 \%)$ and 7 to $11 \mathrm{mmol} / \mathrm{mol}(0.6$ to $1.0 \%)$, respectively.
\end{abstract}

* Correspondence: andrew.fang.h.s@singhealth.com.sg

${ }^{1}$ SingHealth Polyclinics, SingHealth, 167, Jalan Bukit Merah, Connection One, Tower 5, \#15-10, Singapore P.O. 150167, Singapore

Full list of author information is available at the end of the article

C The Author(s). 2022 Open Access This article is licensed under a Creative Commons Attribution 4.0 International License, which permits use, sharing, adaptation, distribution and reproduction in any medium or format, as long as you give appropriate credit to the original author(s) and the source, provide a link to the Creative Commons licence, and indicate if changes were made. The images or other third party material in this article are included in the article's Creative Commons licence, unless indicated otherwise in a credit line to the material. If material is not included in the article's Creative Commons licence and your intended use is not permitted by statutory regulation or exceeds the permitted use, you will need to obtain permission directly from the copyright holder. To view a copy of this licence, visit http://creativecommons.org/licenses/by/4.0/ The Creative Commons Public Domain Dedication waiver (http://creativecommons.org/publicdomain/zero/1.0/) applies to the data made available in this article, unless otherwise stated in a credit line to the data. 
Conclusion: The real-world data on Asians with T2DM in this study show that the magnitudes of OAD initiation and dose titration are marginally lower than the results from clinical trials. During shared decision-making in selecting treatment options, the results enable physicians to communicate realistic expectation of the effect of oral medications on the glycemic control of their patients in primary care.

Keywords: Diabetes mellitus, Glycated hemoglobin, Antidiabetic agent, Asian, Primary care

\section{Background}

The global diabetes prevalence was estimated to be $9.3 \%$ (463 million people) in 2019 and is expected to rise to $10.2 \%$ (578 million people) by 2030 [1]. Likewise, the global direct health expenditure of type 2 diabetes mellitus (T2DM) is projected to grow from USD 760 billion in 2019 to USD 850 billion by 2030 [2]. To mitigate the rising health and economic burdens associated with T2DM, clinical guidelines advocate a multifaceted approach, including diet, lifestyle, and medications to achieve disease control, measured using glycated haemoglobin (HbA1c) [3-5].

Under the National Institute for Health and Care Excellence (NICE) guidelines, several classes of oral antidiabetic drugs (OADs) are available for therapeutic treatment, including biguanides, sulphonyurea, dipeptidyl peptidase-4 (DPP-4) inhibitors, and sodium-glucose cotransporter-2 (SGLT-2) inhibitors [3]. While studies have evaluated the effectiveness of these OADs, most were based on Caucasian populations [6-8]. Asians have different vascular risk profiles from Caucasians $[9,10]$. Literature suggests that due to pathophysiology, clinical presentation, and management, Asians suffer from higher propensity of developing T2DM and faster progression of the disease to complications [11-14]. Given that Asia is the epicenter of T2DM, accounting for $60 \%$ of the world's population with T2DM, it thus becomes critical to understand the effectiveness of OADs in managing T2DM in Asians [15].

As most of the studies evaluating OAD effectiveness were conducted in controlled trial-based settings, it remains uncertain if the magnitude of HbA1c lowering differ in actual clinical practice due to suboptimal medication adherence, psychosocial profiles, lifestyle, and health-seeking behaviour [6-8]. Furthermore, data on OAD up-titration, downtitration, and discontinuation is currently lacking. T2DM is a chronic disease which often requires serial medication titrations to maintain glycemic control over the course of a person's lifetime. This study aims to determine the HbA1c change following titration of various OADs from real-world data among Asians with T2DM managed in primary care. Understanding the impact of various OAD dosage titrations on HbA1c would enable physicians to provide clear expected outcomes of their recommended OAD changes to facilitate better shared, and more informed, decisionmaking with patients.

\section{Methods}

\section{Study design, setting, and population}

A retrospective cohort study was conducted using patient electronic medical records (EMR) from a network of eight polyclinics located in Singapore. These polyclinics manage about 1.8 million patient attendances annually and serves about 1.5 million multi-ethnic Asians (76.2\% Chinese, $15.0 \%$ Malays, $7.4 \%$ Indians, $1.4 \%$ minority ethnic groups) living in the eastern region of Singapore [16]. About one third of patients who attend the polyclinics are aged 65 years and above.

Based on local clinical practice guidelines, patients with T2DM are reviewed by the physicians and nurse physicians with a HbA1c test performed at the in-house laboratory to assess their diabetic control once every 3 to 6 months, with flexibility for closer monitoring if their medical conditions are unstable [5]. Their demographic, clinical, and laboratory information are documented in the polyclinic EMR system.

The study population comprised multi-ethnic adult patients, aged 21 years or older, with diabetic-related diagnoses entered in the EMR (Table 1). Patients with type 1 diabetes were excluded. The clinical data of the study population were extracted from the EMR from January 1, 2015, to December 31, 2019.

\section{Data definition and processing}

Nine different types of OADs in the polyclinic drug formulary were examined for their clinical effectiveness. These OADS are metformin, glipizide, gliclazide, tolbutamide, sitagliptin, linagliptin, dapagliflozin, empagliflozin, and acarbose. The total daily dosage for each OAD was also computed. For example, if a patient was taking metformin $500 \mathrm{mg}$ twice daily, it would be converted to a dose of metformin $1000 \mathrm{mg}$. In selected atypical cases when some patients were prescribed variable OAD doses across different days of the week, the mean daily dose over a week was used to compute the OAD dose.

Patients with fewer than two HbA1c values were excluded as at least two HbA1c values were needed for comparison of OAD effect. Patients who were not on OAD throughout the study period were grouped into the "No OAD" cohort. For the remaining patients, OAD titration was determined by analyzing their prescription records. The records were sorted by patient identifier 
Table 1 Diabetic-related diagnoses, with ICD-10 codes, used to identify eligible patients

\begin{tabular}{ll}
\hline Diabetic-related diagnosis & $\begin{array}{l}\text { ICD-10 } \\
\text { code }\end{array}$ \\
\hline Type 2 diabetes without complication & E11.9 \\
Type 2 diabetes with incipient diabetic nephropathy & E11.21 \\
Type 2 diabetes with established diabetic nephropathy & E11.22 \\
Type 2 diabetes with unspecified neuropathy & E11.40 \\
Unspecified diabetes mellitus with background retinopathy & E14.31 \\
$\begin{array}{ll}\text { Unspecified diabetes mellitus with foot ulcer due to } \\
\text { multiple causes }\end{array}$ & E14.73 \\
\hline
\end{tabular}

Diabetic-related diagnoses based on a restricted set of ICD-10 diagnosis codes used in the polyclinic electronic medical records system. Abbreviations: ICD-10 International Classification of Diseases, 10th Revision

and then by prescription date. Next, the time interval between two consecutive prescriptions for each patient were assessed for OAD discontinuation. A patient was considered to have discontinued taking OAD if there was no record of OAD within a year of the last prescription. The difference in OAD dose between two consecutive prescriptions indicate $\mathrm{OAD}$ dose titrations. Only pairs of consecutive prescriptions with dose titrations were selected for analysis. Patients without such pair of prescriptions were grouped into the "Non-titrators" cohort, while the rest were grouped into the "Titrators" cohort. Patients in these two cohorts could be on monotherapy or a combination of OADs or insulin. The different types of insulin (e.g., detemir, glargine) were combined into a single insulin group in the analysis.

The investigator first identified and collated all pairs of index prescriptions in the "Titrators' cohort". The unique sets of OAD titrations, which consisted of a corresponding pair of initial OAD dose and new OAD dose, were then extracted. An example of an OAD titration is metformin 500 to $1000 \mathrm{mg}$. For each unique OAD titration, the pre-HbA1c indicated the latest glycemic control within 1 year before the OAD dose adjustment and the post-HbA1c reflected the glycemic control beyond 12 weeks to 1 year after the OAD titration. The HbA1c values measured within 12 weeks of titration of an OAD were excluded. This 12-week window was used as HbA1c values are derived from irreversible glucose binding on erythrocytes which have a lifespan of about 90 days, thus reflecting glycemic control over the past 3 months $[17,18]$. It is also consistent with clinical trials for OAD typically have a minimum 12-week follow-up to assess HbA1c lowering efficacy [6]. The 1 year limitation concurred with the recommendation to perform panel tests annually for patients with T2DM based on local clinical practice guidelines [5].

Since patients could have multiple OAD titrations, a HbA1c value could serve both as the post-HbAlc for one titration, as well as the pre-HbA1c for a subsequent titration (Fig. 1). In order to isolate the HbA1c change to each individual OAD titration, we excluded instances where there were multiple titrations (of the OAD of interest, other OADs or insulin) within the 1-year period after the OAD titration of interest.

For those in the "Non-titrators" and "No OAD" cohorts, each of their serial HbA1c values were collated and used as a comparison group. Similar to the "Titrators" cohort, pairs of consecutive HbA1c values with a minimum 12-week interval were used to correlate with the dose adjustment of the respective OAD.

Overall, the mean difference and 95\% confidence interval (CI) between the pre-HbA1c and the post-HbA1c pairs were used to report the effect of OAD up-titration, down-titration, and no-titration. The descriptive analyses which included identifying the pairs, computing the difference in each pre-HbA1c and post-HbA1c pair and then aggregating the mean for each type of OAD titration was performed using the Python "statsmodel" package, version 0.12.1. To further investigate and adjust for the effect of covariates available in the dataset, a multivariate regression analysis was performed, using a multivariate linear regression model adjusting for covariates at each OAD titration. The adjusted change in HbA1c is the least-squares mean value of changes in $\mathrm{HbA1c}$ obtained from multivariable linear regression model. Missing data from biomarkers namely blood pressure readings $(<1 \%)$, body mass index $(10 \%)$, and lipid data (30\%) were handled by imputing the population mean before fitting to the regression model. The rest of the covariates did not have missing values.

\section{Results}

A total of 57,910 unique adult patients with T2DM were extracted from the polyclinic EMR. 53,897 of them (93.1\%) had at least two HbA1c values over the study period. Cohorts of 6535, 3784, and 34,978 patients in the "No OAD," "Non-titrators," and "Titrators" categories, respectively, were identified with at least one pre- and post-HbA1c pair. Analyses were conducted on 54,744, 32,262 , and 77016 HbA1c pairs in the "No OAD," "Nontitrators," and "Titrators" cohorts respectively. Figure 2 shows the derivation of the three patient cohorts.

The baseline characteristics of the patients in each cohort are shown in Table 2. The mean age ranges from 64.0 to 71.9 years across the three cohorts, with slight female predominance $(51.4-57.1 \%)$. The majority of patients in each cohort had Hypertension (87.5-91.0\%) and Dyslipidemia (93.5-94.0\%). In the "Titrators" group, most of the patients had T2DM for at least 3 years (53.8\%).

\section{Change in $\mathrm{HbA1c}$ following OAD titration}

There were a total of 206 unique sets of OAD dosage titrations, for the 9 different OADs-metformin $(n=72)$, 


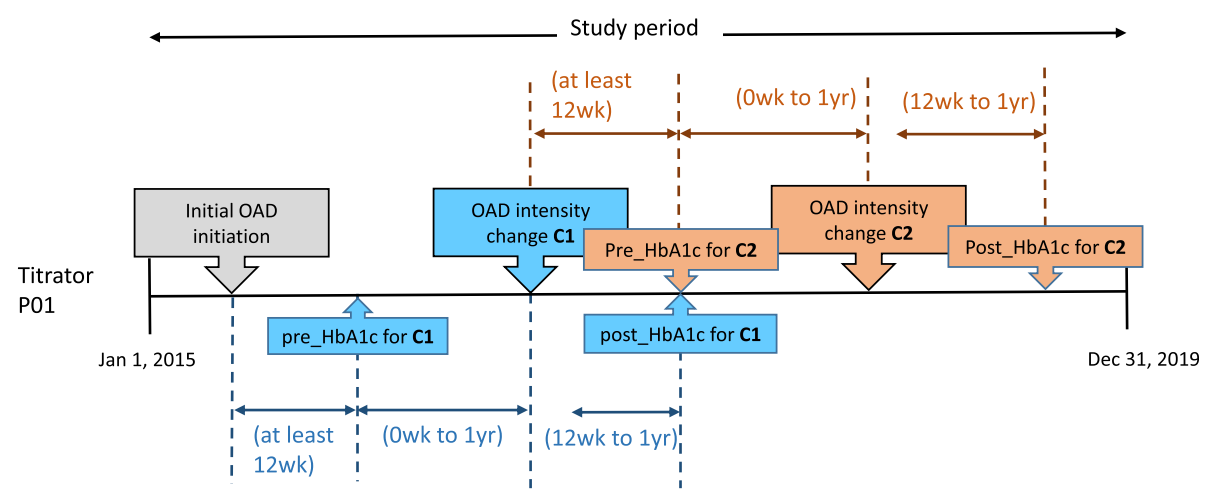

Fig. 1 This figure illustrates the data processing to identify HbA1c pairs for patients in "Titrators" group. To obtain the HbA1c value before OAD titration (pre_HbA1c) and after OAD titration (post_HbA1c) for the analysis, an OAD titration is first identified. In the figure, two OAD titrations (C1 and $\mathrm{C} 2$ ) were identified for the patient (P01). For each OAD titration, the pre_HbA1c is taken to be the most recent $\mathrm{HbA1C}$ result within one year before an $\mathrm{OAD}$ titration, while the post_HbA1c is the first $\mathrm{HbA1} \mathrm{c}$ value within twelve weeks to one year after the OAD titration. Abbreviations: $\mathrm{C} 1=$ first $\mathrm{OAD}$ titration, $\mathrm{C} 2$ = second OAD titration, P01 = illustrative patient, pre_HbA1c = HbA1c before OAD titration; post_HbA1c = HbA1c after the OAD titration

glipizide $(n=41)$, gliclazide $(n=22)$, tolbutamide $(n=24)$, sitagliptin $(n=12)$, linagliptin $(n=6)$, dapagliflozin $(n=8)$, empagliflozin $(n=2)$, and acarbose $(n=19)$. The mean difference in HbA1c for the various OAD titrations are shown in Tables 3, 4, 5, 6, 7, 8, 9, 10, 11. An executive summary of the results illustrating the more typical OAD titrations is found in Fig. 3. For those more familiar with HbA1c results in the National Glycohemoglobin Standardization Program (NGSP) network (represented in \%), the same figure and tables with NGSP units can be found in the supplementary file as Additional file 1: Figure S1 and Additional file 1: Tables S1a to S1i.

Initiation of OADs resulted in a lowering of $\mathrm{HbA} 1 \mathrm{c}$ by 3 to $12 \mathrm{mmol} / \mathrm{mol}$ (0.3 to $1.1 \%)$. Among the instances of
OAD initiation, most commonly started OAD dosages were metformin 500mg $(m=1849)$, glipizide $5 \mathrm{mg}(m=$ $1283)$, and linagliptin $(m=1807)$.

Up-titration of OAD resulted in a mean difference of -1 to $-8 \mathrm{mmol} / \mathrm{mol}(-0.1$ to $-0.7 \%)$ in HbA1c, while down-titration resulted in a mean difference of +1 to +7 $\mathrm{mmol} / \mathrm{mol}(+0.1$ to $+0.6 \%)$. Discontinuation of OADs resulted in an increase of HbA1c by 2 to $11 \mathrm{mmol} / \mathrm{mol}$ $(+0.2$ to $+1.0 \%)$.

Among the various OADs, metformin had the largest overall cumulative HbA1c reduction from initiation to maximum dose -23 to $-32 \mathrm{mmol} / \mathrm{mol}(-2.1$ to $-2.9 \%)$. The sulphonylurea group (glipizide, gliclazide, and tolbutamide) had the largest $\mathrm{HbA1c}$ reduction on initiation

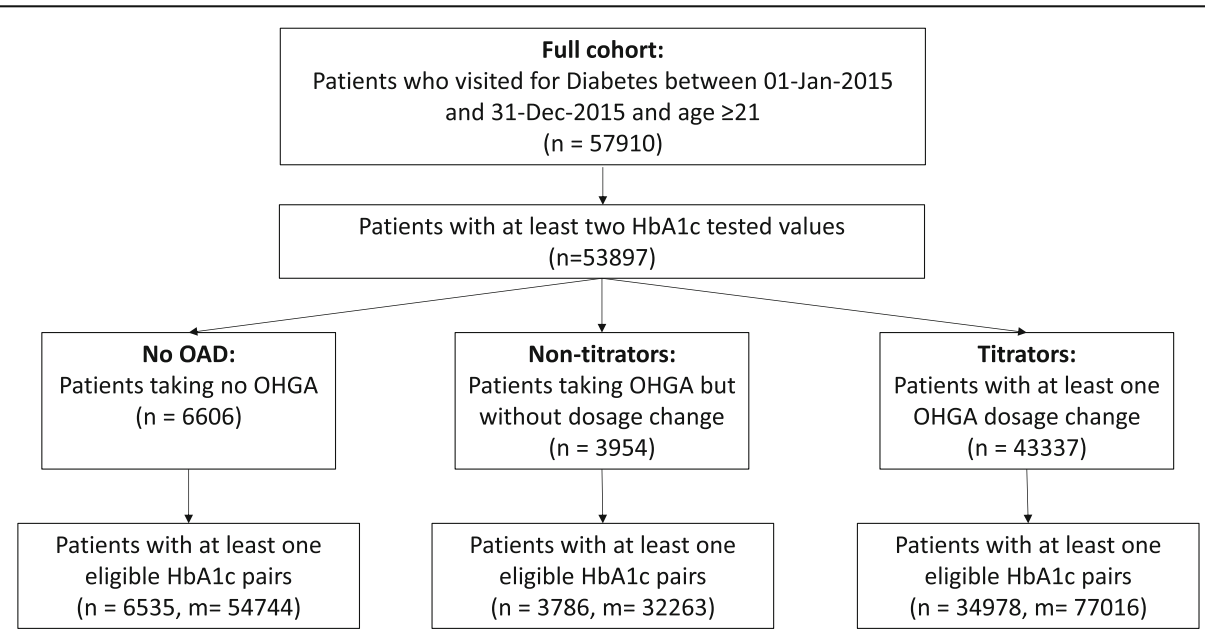

( $n$ : number of patients, m: number of $\mathrm{HbA1c}$ pairs)

Fig. 2 Flow chart illustrating the derivation of the patient cohorts. "No OAD" cohort refers to patients who were not on OAD throughout the study period. "Non-titrators" cohort refers to patients who were on at least one OAD but did not have any OAD dose adjustment during the study period. "Titrators" cohort refers to patients who were on at least one OAD and had at least one OAD dose adjustment during the study period. Abbreviation: $\mathrm{n}=$ number of patients, $\mathrm{m}=$ number of $\mathrm{HbA} 1 \mathrm{c}$ pairs 
Table 2 Baseline characteristics of patients in study cohort

\begin{tabular}{|c|c|c|c|c|}
\hline Characteristics & $\begin{array}{l}\text { No OAD } \\
(n=6535)\end{array}$ & $\begin{array}{l}\text { Non-titrators } \\
(n=3786)\end{array}$ & $\begin{array}{l}\text { Titrators } \\
(n=34978)\end{array}$ & $\begin{array}{l}\text { Full cohort } \\
(n=57910)\end{array}$ \\
\hline Total patients, $n(\%)$ & $6535(100)$ & $3786(100)$ & $34978(100)$ & $57910(100)$ \\
\hline Age (year), mean (SD) & $71.9(11.0)$ & $68.6(10.9)$ & $64.0(10.9)$ & $65.4(11.8)$ \\
\hline Sex, males, $n(\%)$ & $2801(42.9)$ & $1788(47.2)$ & $16997(48.6)$ & $28107(48.5)$ \\
\hline \multicolumn{5}{|l|}{ Race, $n(\%)$} \\
\hline Chinese & $5396(82.6)$ & $2876(76.0)$ & $24530(70.1)$ & $41036(70.9)$ \\
\hline Malay & $600(9.2)$ & $471(12.4)$ & $5478(15.7)$ & $8741(15.1)$ \\
\hline Indian & $339(5.2)$ & $284(7.5)$ & $3448(9.9)$ & $5525(9.5)$ \\
\hline Others & $200(3.1)$ & $155(4.1)$ & $1549(4.4)$ & $2608(4.5)$ \\
\hline Body mass index $\left(\mathrm{kg} / \mathrm{m}^{2}\right)$, mean (SD) & $25.4(4.4)$ & $25.9(4.6)$ & $26.7(4.8)$ & $26.5(4.8)$ \\
\hline \multicolumn{5}{|l|}{ Diagnosis, $n(\%)$} \\
\hline Dyslipidemia & $6109(93.5)$ & 3559(94.0) & 32893(94.0) & 53609(92.6) \\
\hline Hypertension & 5944(91.0) & 3393(89.6) & $30620(87.5)$ & $50483(87.2)$ \\
\hline \multicolumn{5}{|l|}{ Years with diabetes at base visit, $n(\%)$} \\
\hline 0 & $5473(83.7)$ & $279(7.4)$ & $5190(14.8)$ & $14625(25.3)$ \\
\hline 1 & $79(1.2)$ & $422(11.1)$ & $3031(8.7)$ & $4731(8.2)$ \\
\hline 2 & $150(2.3)$ & $430(11.4)$ & $3120(8.9)$ & $4859(8.4)$ \\
\hline 3 & $633(9.7)$ & $2494(65.9)$ & $22008(62.9)$ & $31137(53.8)$ \\
\hline 4 & $63(1.0)$ & $53(1.4)$ & $448(1.3)$ & $715(1.2)$ \\
\hline$\geq 5$ & $137(2.1)$ & $108(2.9)$ & $1182(3.4)$ & $1843(3.2)$ \\
\hline Number of $\mathrm{HbA} 1 \mathrm{c}$ tests per year, mean (SD) & $2.1(0.9)$ & $2.2(1.1)$ & 3.2(0.9) & $2.8(1.2)$ \\
\hline \multicolumn{5}{|l|}{ Number of OAD prescribed, $n(\%)$} \\
\hline 0 & $6535(100.0)$ & $9(0.2)$ & $3737(10.7)$ & $13176(22.8)$ \\
\hline 1 & $0(0.0)$ & $2685(70.9)$ & $13234(37.8)$ & $20517(35.4)$ \\
\hline 2 & $0(0.0)$ & $949(25.1)$ & $13741(39.3)$ & $18776(32.4)$ \\
\hline 3 & $0(0.0)$ & $129(3.4)$ & $3745(10.7)$ & $4782(8.3)$ \\
\hline 4 & $0(0.0)$ & $14(0.4)$ & $521(1.5)$ & $659(1.1)$ \\
\hline On insulin, $n(\%)$ & $0(0.0)$ & $37(1.0)$ & $3868(11.1)$ & $5538(9.6)$ \\
\hline \multicolumn{5}{|l|}{ HbA1c group, $n(\%)$} \\
\hline$<7.0$ & $5735(87.8)$ & $2627(69.4)$ & $13886(39.7)$ & $26901(46.5)$ \\
\hline $7.0-7.9$ & $252(3.9)$ & $852(22.5)$ & $11425(32.7)$ & $15299(26.4)$ \\
\hline $8.0-8.9$ & $15(0.2)$ & $124(3.3)$ & $4292(12.3)$ & $5722(9.9)$ \\
\hline $9.0-9.9$ & $1(0.0)$ & $29(0.8)$ & $1771(5.1)$ & $2560(4.4)$ \\
\hline$\geq 10$ & $1(0.0)$ & $38(1.0)$ & $1828(5.2)$ & $2961(5.1)$ \\
\hline Missing ${ }^{1}$ & $531(8.1)$ & $116(3.1)$ & $1776(5.1)$ & $4467(7.7)$ \\
\hline Number of OAD prescriptions per year, mean (SD) & $0.0(0.0)$ & $2.2(1.3)$ & $3.5(1.2)$ & $3.1(1.5)$ \\
\hline
\end{tabular}

${ }^{1}$ These patients did not have a HbA1c test done at their baseline visit. Abbreviations: HbA1c glycated hemoglobin, OAD oral anti-diabetic drug, $S D$ standard deviation

of 11 to $12 \mathrm{mmol} / \mathrm{mol}(-1.0$ to $-1.1 \%)$. The newer OAD groups such as DPP-4 inhibitors and SGLT-2 inhibitors, represented by sitagliptin and linagliptin; and dapagliflozin and empagliflozin, respectively, had a more modest HbA1c lowering effect on initiation of 8 to $10 \mathrm{mmol} /$ $\mathrm{mol}(0.7$ to $0.9 \%)$ and 7 to $11 \mathrm{mmol} / \mathrm{mol}(0.6$ to $1.0 \%)$, respectively. Acarbose had the lowest $\mathrm{HbA1c}$ reduction on initiation of 4 to $7 \mathrm{mmol} / \mathrm{mol}(0.4$ to $0.6 \%)$ and on up-titration of 1 to $2 \mathrm{mmol} / \mathrm{mol}$ (0.1 to $0.2 \%)$.

For comparison, among the HbA1c pairs from patients in the "No OAD" and "Non-titrators" cohort, no significant HbA1c change was noted in these two groups. Detailed results from this analysis can be found in the Additional file 2: Table S1. Multivariate regression to 
Table 3 Change in $\mathrm{HbA} 1 \mathrm{c}$ values (with $\mathrm{HbA} 1 \mathrm{c}$ denoted in $\mathrm{mmol} / \mathrm{mol}$ ) after metformin titration

\begin{tabular}{|c|c|c|c|c|c|c|c|c|c|c|c|c|}
\hline \multicolumn{13}{|c|}{ Metformin dose after titration } \\
\hline 0 & 125 & 250 & 500 & 750 & 850 & 1000 & 1500 & 1700 & 2000 & 2250 & 2550 & 3000 \\
\hline & & $\begin{array}{l}-3(-4, \\
-3) \# m= \\
487\end{array}$ & $\begin{array}{l}-7(-7, \\
-7) \# m= \\
1849\end{array}$ & & & $\begin{array}{l}-10(-11 \\
-9) \# m=676\end{array}$ & $\begin{array}{l}-8(-12 \\
-3) \# m=36\end{array}$ & $\begin{array}{l}-7(-11 \\
-3) \# m=60\end{array}$ & & & & \\
\hline & & $\begin{array}{l}-4(-7, \\
-2) \# m=34\end{array}$ & & & & & & & & & & \\
\hline $\begin{array}{l}\text { 6(4, } \\
8) \# \mathrm{~m}= \\
173\end{array}$ & $\begin{array}{l}\text { 2(1, } \\
\text { 3) \#m= } \\
105\end{array}$ & & $\begin{array}{l}-4(-6, \\
-4) \# m= \\
906\end{array}$ & & & $\begin{array}{l}-10(-13 \\
-8) \# m=87\end{array}$ & & & & & & \\
\hline \multirow[t]{3}{*}{$\begin{array}{l}6(4, \\
8) \# m= \\
220\end{array}$} & & $\begin{array}{l}3(3, \\
4) \# m= \\
1412\end{array}$ & & $\begin{array}{l}-3(-4, \\
-2) \# m= \\
282\end{array}$ & & $\begin{array}{l}-7(-7, \\
-7) \# m= \\
3108\end{array}$ & $\begin{array}{l}-9(-12 \\
-6) \# m=77\end{array}$ & $\begin{array}{l}-14(-18 \\
-11) \# m=53\end{array}$ & & & & \\
\hline & & & $\begin{array}{l}\text { 2(1, } \\
\text { 3) } \# \mathrm{~m}=182\end{array}$ & & & $\begin{array}{l}-3(-4 \\
-2) \# m=225\end{array}$ & $\begin{array}{l}-7(-9 \\
-6) \# m= \\
190\end{array}$ & $\begin{array}{l}-8(-12 \\
-4) \# m=31\end{array}$ & & & & \\
\hline & & & $\begin{array}{l}\text { 3(-0, } \\
6) \# m=34\end{array}$ & & & $\begin{array}{l}\text { 1(-1, } \\
\text { 3) \#m=58 }\end{array}$ & & $\begin{array}{l}-3(-6, \\
-2) \# m=161\end{array}$ & & & $\begin{array}{l}-2(-6, \\
0) \# m=39\end{array}$ & \\
\hline $\begin{array}{l}8(4, \\
10) \# m= \\
137\end{array}$ & & $\begin{array}{l}8(3, \\
12) \# \mathrm{~m}= \\
31\end{array}$ & $\begin{array}{l}\text { 6(4, } \\
6) \# \mathrm{~m}= \\
1953\end{array}$ & $\begin{array}{l}-1(-2, \\
0) \# \mathrm{~m}= \\
105\end{array}$ & $\begin{array}{l}-1(-3, \\
0) \# m= \\
57\end{array}$ & & $\begin{array}{l}-4(-6, \\
-5) \# m= \\
2150\end{array}$ & $\begin{array}{l}-7(-7, \\
-6) \# \mathrm{~m}= \\
1670\end{array}$ & $\begin{array}{l}-4(-7 \\
-3) \# \mathrm{~m}= \\
198\end{array}$ & & $\begin{array}{l}-10(-14 \\
-7) \# m=47\end{array}$ & $\begin{array}{l}-6(-8, \\
-2) \# m=47\end{array}$ \\
\hline $\begin{array}{l}\text { 12(7, } \\
\text { 16) \#m= } \\
41\end{array}$ & & & $\begin{array}{l}8(5, \\
11) \# m=96\end{array}$ & $\begin{array}{l}\text { 3(1, } \\
6) \# m=91\end{array}$ & & $\begin{array}{l}4(3,4) \# \mathrm{~m}= \\
1128\end{array}$ & & $\begin{array}{l}-3(-3, \\
-2) \# m=739\end{array}$ & $\begin{array}{l}-4(-6 \\
-3) \# m= \\
418\end{array}$ & $\begin{array}{l}-7(-7, \\
6) \# m= \\
498\end{array}$ & $\begin{array}{l}-8(-9, \\
-7) \# m= \\
398\end{array}$ & \\
\hline \multirow[t]{5}{*}{$\begin{array}{l}\text { 13(8), } \\
19) \# m= \\
46\end{array}$} & & & $\begin{array}{l}8(3, \\
12) \# m=38\end{array}$ & & $\begin{array}{l}2(1 \\
3) \# \mathrm{~m}= \\
240\end{array}$ & $\begin{array}{l}6(6,7) \# \mathrm{~m}= \\
773\end{array}$ & $\begin{array}{l}1(0, \\
2) \# m=142\end{array}$ & & $\begin{array}{l}-2(-3, \\
-2) \# \mathrm{~m}= \\
611\end{array}$ & & $\begin{array}{l}-6(-6, \\
-4) \# m= \\
1154\end{array}$ & \\
\hline & & & & & & $\begin{array}{l}4(3,6) \# \mathrm{~m}= \\
228\end{array}$ & $\begin{array}{l}3(1 \\
6) \# m=82\end{array}$ & $\begin{array}{l}3(1,4) \# \mathrm{~m}= \\
127\end{array}$ & & & $\begin{array}{l}-3(-4, \\
-2) \# m= \\
179\end{array}$ & $\begin{array}{l}-3(-6 \\
-2) \# m= \\
458\end{array}$ \\
\hline & & & & & & $\begin{array}{l}6(2,9) \# m= \\
34\end{array}$ & $\begin{array}{l}\text { 3(3, } \\
\text { 4) } \# m=227\end{array}$ & $\begin{array}{l}\text { 1(-2, } \\
3) \# m=30\end{array}$ & $\begin{array}{l}\text { 1(-2, } \\
\text { 3) } \# \mathrm{~m}=42\end{array}$ & & $\begin{array}{l}-4(-4, \\
-3) \# m= \\
311\end{array}$ & $\begin{array}{l}-4(-7 \\
-2) \# m= \\
124\end{array}$ \\
\hline & & & & & $\begin{array}{l}3(0, \\
7) \# m= \\
30\end{array}$ & $\begin{array}{l}6(3,9) \# m= \\
75\end{array}$ & $\begin{array}{l}\text { 6(4, } \\
\text { 7) } \# m=165\end{array}$ & $\begin{array}{l}3(3,4) \# \mathrm{~m}= \\
920\end{array}$ & $\begin{array}{l}-1(-2, \\
0) \# m= \\
182\end{array}$ & $\begin{array}{l}3(-0 \\
6) \# m=33\end{array}$ & & $\begin{array}{l}-3(-4 \\
-2) \# m= \\
642\end{array}$ \\
\hline & & & & & & $\begin{array}{l}\text { 10(6, } \\
\text { 14)\#m=41 }\end{array}$ & $\begin{array}{l}9(6, \\
11) \# m=65\end{array}$ & & $\begin{array}{l}4(3 \\
4) \# m= \\
438\end{array}$ & $\begin{array}{l}\text { 4(2, } \\
\text { 7) } \# m=35\end{array}$ & $\begin{array}{l}2(0, \\
3) \# m=129\end{array}$ & \\
\hline
\end{tabular}

Change in HbA1c values after metformin initiation, titration, or discontinuation. The values above the diagonal represent the instances where the medication has been initiated or up-titrated, while the values below the diagonal represent instances where the medication has been down-titrated or discontinued. The values refer to the mean difference in $\mathrm{HbA1c}(\mathrm{MD})$ and $95 \%$ confidence intervals. MD below 0 indicate a lowering in $\mathrm{HbA} 1 \mathrm{c}$ while those above 0 indicate an increase in $\mathrm{HbA1c}$ \#m refers to the number of $\mathrm{HbA1c}$ pairs for that dose titration

adjust for covariates which include gender, race, number of OADs prescribed, insulin use, and time to follow-up revealed that baseline $\mathrm{HbA1c}$ and insulin use had the largest impact of 2 to $4 \mathrm{mmol} / \mathrm{mol}(0.2$ to $0.4 \%)$. A visualization of the covariate weights and the adjusted results can be found in the Additional file 2: Fig S1, Additional file 2: Table S2 and Additional file 2: Tables S3a to S3i.

\section{Discussion}

The study enhances our understanding of the effect size resultant from various $\mathrm{OAD}$ dose titrations on $\mathrm{HbA1c}$ beyond trial-based studies. The latter tends to focus on regimental dose initiations or up-titrations [6-8]. Furthermore, findings from our study concur with the systematic review, which reports that OAD up-titration results in lower effect on $\mathrm{HbA1c}$ compared to OAD initiation [6]. In this study, any OAD initiation for an Asian patient with T2DM does not result in the expected magnitude of $\mathrm{HbA} 1 \mathrm{c}$ reduction, being 3 to $12 \mathrm{mmol} / \mathrm{mol}$ (0.3 to $1.1 \%$ ) lower compared to results from clinical trials [19-21]. A review of clinical trials published by Sherifali et al. found that most OADs lowered HbA1c levels by 6 to $14 \mathrm{mmol} / \mathrm{mol}$ ( 0.5 to $1.25 \%$ ) [6]. One possible reason for this discrepancy is due to suboptimal medication adherence among patients in the real world [22]. 
Table 4 Change in HbA1c values (with HbA1c denoted in mmol/mol) after glipizide titration

\begin{tabular}{|c|c|c|c|c|c|c|c|c|c|c|}
\hline \multicolumn{11}{|c|}{ Glipizide dose after titration } \\
\hline 0 & 2.5 & 5 & 7.5 & 10 & 12.5 & 15 & 20 & 25 & 30 & 40 \\
\hline & $\begin{array}{l}-8(-9, \\
-7) \# m=471\end{array}$ & $\begin{array}{l}-11(-12 \\
-10) \# m=1283\end{array}$ & & $\begin{array}{l}-13(-14 \\
-11) \# m=364\end{array}$ & & & $\begin{array}{l}-1(-7, \\
3) \# m=41\end{array}$ & & & \\
\hline $\begin{array}{l}8(8,9) \# \mathrm{~m}= \\
596\end{array}$ & & $\begin{array}{l}-6(-6,-4) \# \mathrm{~m}= \\
660\end{array}$ & & $\begin{array}{l}-9(-14,-3) \# m= \\
51\end{array}$ & & & & & & \\
\hline \multirow[t]{2}{*}{$\begin{array}{l}\text { 10(10, } \\
\text { 11) } \# \mathrm{~m}=818\end{array}$} & $\begin{array}{l}4(3,4) \# \mathrm{~m}= \\
815\end{array}$ & & $\begin{array}{l}-4(-7 \\
-1) \# m=82\end{array}$ & $\begin{array}{l}-3(-3,-2) \# \mathrm{~m}= \\
1686\end{array}$ & & & & & & \\
\hline & & $2(0,6) \# m=59$ & & $\begin{array}{l}-0(-2,1) \# m= \\
157\end{array}$ & & $\begin{array}{l}-0(-1, \\
\text { 1) } \# m=133\end{array}$ & & & & \\
\hline \multirow[t]{3}{*}{$\begin{array}{l}\text { 10(9, } \\
\text { 12) } \# \mathrm{~m}=260\end{array}$} & $\begin{array}{l}3(1,6) \# \mathrm{~m}= \\
32\end{array}$ & $\begin{array}{l}3(3,3) \# \mathrm{~m}= \\
1042\end{array}$ & $\begin{array}{l}0(-1 \\
1) \# m=267\end{array}$ & & & $\begin{array}{l}-2(-2 \\
-1) \# m=966\end{array}$ & $\begin{array}{l}-2(-3, \\
-2) \# m=753\end{array}$ & & & \\
\hline & & & & & & & & $\begin{array}{l}0(-2,2) \# \mathrm{~m}= \\
57\end{array}$ & & \\
\hline & & $1(-1,3) \# m=30$ & $\begin{array}{l}1(0,3) \# \mathrm{~m}= \\
50\end{array}$ & $\begin{array}{l}2(1,3) \# m= \\
376\end{array}$ & & & $\begin{array}{l}-1(-1, \\
0) \# m=852\end{array}$ & & $\begin{array}{l}-1(-2, \\
\text { 1) } \# \mathrm{~m}=294\end{array}$ & \\
\hline \multirow[t]{2}{*}{$\begin{array}{l}\text { 10(6, } \\
\text { 13) } \# \mathrm{~m}=47\end{array}$} & & & & $\begin{array}{l}2(2,3) \# \mathrm{~m}= \\
514\end{array}$ & $\begin{array}{l}-1(-2, \\
0) \# m=145\end{array}$ & $\begin{array}{l}0(0,1) \# \mathrm{~m}= \\
362\end{array}$ & & $\begin{array}{l}-2(-3 \\
-1) \# m=617\end{array}$ & $\begin{array}{l}-1(-2, \\
0) \# \mathrm{~m}=465\end{array}$ & \\
\hline & & & & & & $\begin{array}{l}0(-2,2) \# m= \\
72\end{array}$ & $\begin{array}{l}1(-1,2) \# \mathrm{~m}= \\
95\end{array}$ & & $\begin{array}{l}-1(-2, \\
0) \# \mathrm{~m}=444\end{array}$ & \\
\hline $\begin{array}{l}7(2,10) \# \mathrm{~m}= \\
45\end{array}$ & & & & $2(-1,4) \# m=68$ & & $\begin{array}{l}3(2,4) \# \mathrm{~m}= \\
259\end{array}$ & $\begin{array}{l}3(2,4) \# \mathrm{~m}= \\
323\end{array}$ & $\begin{array}{l}2(0,3) \# \mathrm{~m}= \\
83\end{array}$ & & $\begin{array}{l}-3(-6 \\
0) \# m=73\end{array}$ \\
\hline
\end{tabular}

Change in HbA1c values after glipizide initiation, titration or discontinuation. The values above the diagonal represent the instances where the medication has been initiated or up-titrated, while the values below the diagonal represent instances where the medication has been down-titrated or discontinued. The values refer to the mean difference in $\mathrm{HbA} 1 \mathrm{C}(\mathrm{MD})$ and $95 \%$ confidence intervals. MD below 0 indicate a lowering in $\mathrm{HbA} 1 \mathrm{c}$ while those above 0 indicate an increase in $\mathrm{HbA1c}$. \#m refers to the number of $\mathrm{HbA1c}$ pairs for that dose titration

Table 5 Change in $\mathrm{HbA} 1 \mathrm{c}$ values (with $\mathrm{HbA} 1 \mathrm{c}$ denoted in $\mathrm{mmol} / \mathrm{mol}$ ) after gliclazide titration

\begin{tabular}{|c|c|c|c|c|c|c|c|c|c|}
\hline \multicolumn{10}{|c|}{ Gliclazide dose after titration } \\
\hline 0 & 30 & 40 & 60 & 80 & 90 & 120 & 160 & 240 & 320 \\
\hline & $\begin{array}{l}-10(-13 \\
-8) \# m=57\end{array}$ & $\begin{array}{l}-12(-14 \\
-10) \# m=89\end{array}$ & & $\begin{array}{l}-12(-14 \\
-11) \# m=174\end{array}$ & & & & & \\
\hline $\begin{array}{l}8(4,10) \# m= \\
44\end{array}$ & & & $\begin{array}{l}-2(-4, \\
0) \# m=73\end{array}$ & & & & & & \\
\hline \multirow[t]{2}{*}{$\begin{array}{l}\text { 13(10, } \\
15) \# m=108\end{array}$} & & & & $\begin{array}{l}-6(-7,-3) \# m= \\
109\end{array}$ & & & & & \\
\hline & $4(2,6) \# m=49$ & & & & $\begin{array}{l}-3(-7, \\
\text { 1)\#m=38 }\end{array}$ & $\begin{array}{l}-1(-6, \\
3) \# m=33\end{array}$ & & & \\
\hline \multirow[t]{5}{*}{$\begin{array}{l}11(9,13) \# m= \\
91\end{array}$} & & $3(2,4) \# m=171$ & & & & & $\begin{array}{l}-3(-4,-2) \# m= \\
233\end{array}$ & & \\
\hline & & & & & & & & $\begin{array}{l}-3(-6,-1) \# m= \\
34\end{array}$ & \\
\hline & & & & $3(2,4) \# m=172$ & & $\begin{array}{l}-1(-4, \\
\text { 1)\#m=59 }\end{array}$ & & $\begin{array}{l}-3(-6,-1) \# m= \\
113\end{array}$ & $\begin{array}{l}-4(-7,-2) \# m= \\
112\end{array}$ \\
\hline & & & & & & & $\begin{array}{l}1(-2,3) \# m= \\
50\end{array}$ & & $\begin{array}{l}-2(-4,1) \# \mathrm{~m}= \\
77\end{array}$ \\
\hline & & & & & & & $\begin{array}{l}2(1,4) \# \mathrm{~m}= \\
119\end{array}$ & $\begin{array}{l}3(-2,8) \# \mathrm{~m}= \\
31\end{array}$ & \\
\hline
\end{tabular}

Change in HbA1c values after gliclazide initiation, titration, or discontinuation. The values above the diagonal represent the instances where the medication has been initiated or up-titrated, while the values below the diagonal represent instances where the medication has been down-titrated or discontinued. The values refer to the mean difference in $\mathrm{HbA1c}(\mathrm{MD})$ and $95 \%$ confidence intervals. MD below 0 indicate a lowering in $\mathrm{HbA} 1 \mathrm{c}$ while those above 0 indicate an increase in $\mathrm{HbA} 1 \mathrm{c}$. \#m refers to the number of $\mathrm{HbA1c}$ pairs for that dose titration 
Table 6 Change in $\mathrm{HbA1c}$ values (with $\mathrm{HbA} 1 \mathrm{c}$ denoted in $\mathrm{mmol} / \mathrm{mol}$ ) after tolbutamide titration

\begin{tabular}{|c|c|c|c|c|c|c|c|c|}
\hline \multicolumn{9}{|c|}{ Tolbutamide dose after titration } \\
\hline 0 & 250 & 500 & 750 & 1000 & 1500 & 2000 & 2250 & 3000 \\
\hline & $\begin{array}{l}-8(-11,-6) \# m= \\
52\end{array}$ & $\begin{array}{l}-12(-14,-9) \# \mathrm{~m}= \\
103\end{array}$ & & & & & & \\
\hline $6(3,7) \# \mathrm{~m}=90$ & & $-6(-8,-3) \# m=92$ & & & & & & \\
\hline \multirow[t]{2}{*}{$\begin{array}{l}7(6,8) \# \mathrm{~m}= \\
177\end{array}$} & $3(2,4) \# m=243$ & & $\begin{array}{l}-3(-7,0) \# \mathrm{~m}= \\
44\end{array}$ & $\begin{array}{l}-6(-7,-4) \# m= \\
203\end{array}$ & & & & \\
\hline & & $1(-1,2) \# m=89$ & & $1(-3,6) \# m=37$ & $-6(-8,-2) \# m=52$ & & & \\
\hline \multirow[t]{5}{*}{$\begin{array}{l}8(3,11) \# m= \\
45\end{array}$} & & $4(3,4) \# m=277$ & & & $\begin{array}{l}-3(-6,-2) \# m= \\
187\end{array}$ & & & \\
\hline & & & $3(2,6) \# \mathrm{~m}=64$ & $1(0,2) \# m=190$ & & $\begin{array}{l}-1(-4,2) \# m= \\
42\end{array}$ & $\begin{array}{l}-6(-8,-3) \# m= \\
96\end{array}$ & \\
\hline & & & & $7(3,11) \# \mathrm{~m}=50$ & & & & $\begin{array}{l}-7(-11,-3) \# m= \\
42\end{array}$ \\
\hline & & & & & $4(3,6) \# m=95$ & & & $-4(-7,-2) \# m=62$ \\
\hline & & & & & $6(2,9) \# m=43$ & $3(2,6) \# m=67$ & & \\
\hline
\end{tabular}

Change in HbA1c values after tolbutamide initiation, titration, or discontinuation. The values above the diagonal represent the instances where the medication has been initiated or up-titrated, while the values below the diagonal represent instances where the medication has been down-titrated or discontinued. The values refer to the mean difference in $\mathrm{HbA1c}(\mathrm{MD})$ and $95 \%$ confidence intervals. MD below 0 indicate a lowering in $\mathrm{HbA} 1 \mathrm{c}$ while those above 0 indicate an increase in $\mathrm{HbA} 1 \mathrm{c}$. \#m refers to the number of $\mathrm{HbA} 1 \mathrm{c}$ pairs for that dose titration

This is supported by the fact that newer single daily dose of OADs (DPP-4 inhibitors and SGLT-2 inhibitors) achieve comparable $\mathrm{HbA1c}$ reduction with those from clinical trials [23-25]. The simplified dosing of newer OADs is postulated to result in better medication adherence and maintain its effectiveness in real-world setting vis-à-vis to clinical trials [26]. In contrast, patients taking multiple doses of metformin and sulphonylurea are often associated with poorer medication adherence and consequently attain lower HbA1c decline than expected $[19,20]$.

Table 7 Change in HbA1c values (with HbA1c denoted in $\mathrm{mmol} / \mathrm{mol}$ ) after sitagliptin titration

\begin{tabular}{|c|c|c|c|c|}
\hline \multicolumn{5}{|c|}{ Sitagliptin dose after titration } \\
\hline 0 & 25 & 50 & 75 & 100 \\
\hline & $\begin{array}{l}-8(-9,-6) \# m= \\
266\end{array}$ & $\begin{array}{l}-8(-9,-7) \# m= \\
411\end{array}$ & & $\begin{array}{l}-6(-9,-2) \# m= \\
86\end{array}$ \\
\hline $\begin{array}{l}8(6,9) \# m= \\
93\end{array}$ & & $\begin{array}{l}-3(-4,-2) \# m= \\
225\end{array}$ & & \\
\hline \multirow[t]{2}{*}{$\begin{array}{l}8(6,10) \# \mathrm{~m}= \\
156\end{array}$} & $3(0,6) \# m=56$ & & $\begin{array}{l}-3(-7, \\
0) \# m=43\end{array}$ & $\begin{array}{l}-2(-4,-1) \# m= \\
439\end{array}$ \\
\hline & & & & $\begin{array}{l}1(-3,6) \# \mathrm{~m}= \\
35\end{array}$ \\
\hline
\end{tabular}

$$
\text { 7(4, 9)\#m= 2(0,3)\#m=91 }
$$$$
82
$$

Change in HbA1c values after sitagliptin initiation, titration, or discontinuation. The values above the diagonal represent the instances where the medication has been initiated or up-titrated, while the values below the diagonal represent instances where the medication has been down-titrated or discontinued. The values refer to the mean difference in HbA1c (MD) and 95\% confidence intervals. MD below 0 indicate a lowering in $\mathrm{HbA1c}$ while those above 0 indicate an increase in $\mathrm{HbA} 1 \mathrm{c}$. \#m refers to the number of $\mathrm{HbA} 1 \mathrm{c}$ pairs for that dose titration
The OAD dose up-titration effect on $\mathrm{HbA} 1 \mathrm{c}$ reduction of 1 to $8 \mathrm{mmol} / \mathrm{mol}(0.1$ to $0.7 \%)$ in this study is comparable to the $2.6 \mathrm{mmol} / \mathrm{mol}(0.2 \%) \mathrm{HbA} 1 \mathrm{c}$ decline after OAD dose escalation in an American-based real-world study [27]. Genetic variations between Caucasians and Asians and the resultant differences in pharmacokinetic and pharmacodynamics effects of OAD could account for the difference [28]. A trial comparing linagliptin $5 \mathrm{mg}$ dose among Japanese, Asian, and Caucasian patients with T2DM also revealed greater $\mathrm{HbA1c}$ reduction in the Japanese and Asian (non-Japanese) groups relative to those in the Caucasians [29].

The results reveal that cumulative $\mathrm{HbA} 1 \mathrm{c}$ reduction is greater with incremental dose up-titrations rather than a large increase in dose. For example, a dose increase from metformin 0 to $1000 \mathrm{mg}$ resulted in a $0.9 \%$ reduction in

Table 8 Change in $\mathrm{HbA} 1 \mathrm{c}$ values (with $\mathrm{HbA} 1 \mathrm{c}$ denoted in $\mathrm{mmol} / \mathrm{mol}$ ) after linaliptin titration

\begin{tabular}{|c|c|c|}
\hline \multicolumn{3}{|c|}{ Linagliptin dose after titration } \\
\hline 0 & 2.5 & 5 \\
\hline & $-9(-11,-7) \# m=139$ & $-10(-10,-9) \# m=1807$ \\
\hline $6(2,9) \# m=32$ & & $-3(-6,-1) \# m=79$ \\
\hline $7(6,8) \# m=363$ & $2(0,4) \# m=36$ & \\
\hline
\end{tabular}

Change in $\mathrm{HbA1c}$ values after linagliptin initiation, titration, or discontinuation. The values above the diagonal represent the instances where the medication has been initiated or up-titrated, while the values below the diagonal represent instances where the medication has been down-titrated or discontinued. The values refer to the mean difference in $\mathrm{HbA1c}(\mathrm{MD})$ and $95 \%$ confidence intervals. MD below 0 indicate a lowering in $\mathrm{HbA1c}$ while those above 0 indicate an increase in $\mathrm{HbA1c} \# \mathrm{~m}$ refers to the number of $\mathrm{HbA} 1 \mathrm{c}$ pairs for that dose titration 
Table 9 Change in $\mathrm{HbA1c}$ values (with $\mathrm{HbA1c}$ denoted in $\mathrm{mmol} / \mathrm{mol}$ ) after dapagliflozin titration

\begin{tabular}{|c|c|c|c|}
\hline \\
\hline \multicolumn{4}{|c|}{$\begin{array}{lll}\text { Dapagilitiozin dose after titration } \\
0 & 2.5 & 5\end{array}$} \\
\hline & $-9(-11,-6) \# m=67$ & $-9(-10,-8) \# m=570$ & $-11(-11,-10) \# m=722$ \\
\hline & & $-1(-4,2) \# m=39$ & \\
\hline $3(1,7) \# m=85$ & & & $-3(-4,-2) \# m=264$ \\
\hline $7(3,9) \# m=96$ & & $1(-1,3) \# m=76$ & \\
\hline
\end{tabular}

Change in $\mathrm{HbA1c}$ values after dapagliflozin initiation, titration, or discontinuation. The values above the diagonal represent the instances where the medication has been initiated or up-titrated, while the values below the diagonal represent instances where the medication has been down-titrated or discontinued. The values refer to the mean difference in $\mathrm{HbA1c}(\mathrm{MD})$ and $95 \%$ confidence intervals. MD below 0 indicate a lowering in $\mathrm{HbA1c}$ while those above 0 indicate an increase in $\mathrm{HbA1c}$. \#m refers to the number of $\mathrm{HbA1c}$ pairs for that dose titration

HbA1c, while a phased increase from 0 to $500 \mathrm{mg}$, and then from 500 to $1000 \mathrm{mg}$ would have resulted in a 13 $\mathrm{mmol} / \mathrm{mol}(1.2 \%)$ reduction in HbA1c. Patients who are started on higher doses of medications may have lower medication adherence due to concern of adverse effects with higher doses, such as hypoglycemia [30]. Hence, a phased approach to medication up-titration is not only prudent to minimize adverse effects, but also more effective in achieving improved glycemic control.

\section{Study strength and limitations}

Analysis of real-world data of a captive population of Asian patients constitutes a strength in this study. This allows us to account for the effects of real-world practicalities such as suboptimal medication adherence and may be more generalizable to patients in everyday practice. Such real-world evidence have become increasingly important in providing evidence for treatment effectiveness in clinical practice and can complement results from clinical trials in setting more realistic expectations for both physicians and patients on the attainment of HbA1c treatment goals [31].

Another benefit of using real-world data is the opportunity to gain insight on the impact of down-titrating and discontinuing OADs on HbA1c levels. Such changes would be challenging to elucidate from clinical trials due

Table $\mathbf{1 0}$ Change in $\mathrm{HbA} 1 \mathrm{c}$ values (with $\mathrm{HbA} 1 \mathrm{c}$ denoted in $\mathrm{mmol} / \mathrm{mol}$ ) after empagliflozin titration

\begin{tabular}{|c|c|c|}
\hline \multicolumn{3}{|c|}{ Empagliflozin dose after titration } \\
\hline 0 & 12.5 & 25 \\
\hline & $-8(-10,-6) \# m=152$ & $-7(-9,-5) \# m=101$ \\
\hline
\end{tabular}

Change in $\mathrm{HbA} 1 \mathrm{c}$ values after empagliflozin initiation, titration, or discontinuation. The values above the diagonal represent the instances where the medication has been initiated or up-titrated, while the values below the diagonal represent instances where the medication has been down-titrated or discontinued. The values refer to the mean difference in $\mathrm{HbA1c}(\mathrm{MD})$ and 95\% confidence intervals. MD below 0 indicate a lowering in $\mathrm{HbA} 1 \mathrm{c}$ while those above 0 indicate an increase in $\mathrm{HbA1c}$. \#m refers to the number of $\mathrm{HbA1c}$ pairs for that dose titration to protocol design and implementation reasons. Clinical practice is ideally centered on physician-patient shared decision-making in the selection of pharmacotherapy and addressing concerns of treatment options. Intentional dose adjustment is applied on the emergence of known side-effects from the medication. Including allergies and hypoglycemia from OAD. However, unilateral and undisclosed medication dose adjustments seem to be common in clinical practice secondary to a variety of reasons, from patients' perceived adverse effects, related or unrelated to the OAD, to their own volition or ideation without physician input. Such behavior appears prevalent from our study data, with 16,174 instances of OAD down-titration and 4306 instances of OAD discontinuation. These results provide an estimation of magnitude change in glycemic control in the subset of the patients with reduction or discontinuity of their OAD therapy. Nevertheless, the magnitude on HbA1c increase in this study was lower for OAD down-titration and discontinuation than its dose escalation. This may be an avenue for further research into a possible sustained effect of OAD on HbA1c even after dose-reduction or discontinuation.

This study has its limitations. It uses an observational and retrospective study design and cannot account for unobserved heterogeneity (e.g., in diet and exercise, major health events) as would be in a prospective clinical trial. Nevertheless, until a large-scale prospective trial involving OADs an Asian primary care patients, this study will provide the information on the real-world effectiveness of a variety of OADs in this population. Furthermore, such study designs have also been used to generate valuable insights into OAD effectiveness on other populations [32-34]. Another limitation is that various time intervals between OAD titration and HbA1c tests were used (more than 12 weeks for preHbA1c and 12 weeks to 1 year for post-HbA1c). However, this is mitigated by evidence that changes in HbA1c after OAD titration tend to plateau after 12 weeks [35]. While recognizing that the HbA1c lowering effect was lesser in than found in trial-based studies, the latter were centered largely on Caucasian populations. Therefore, the effect sizes of OADs on glycemic control may not be applicable to non-Asians, considering the genetic differences in OAD pharmacology. This study covered only the OADs available in the drug formulary of the institution. Other classes of OAD such as the meglitinide and thiazolidinedione were excluded. The concomitant decreased drug clearance from renal impairment in chronic kidney diseases, drug-drug interactions, and the use of insulin in combination with OADs were not examined on their impact on the results. In order to mitigate some of these limitations, cases with concurrent $\mathrm{OAD}$ titrations were also excluded, such that 
Table 11 Change in $\mathrm{HbA} 1 \mathrm{c}$ values (with $\mathrm{HbA} 1 \mathrm{c}$ denoted in $\mathrm{mmol} / \mathrm{mol}$ ) after acarbose titration

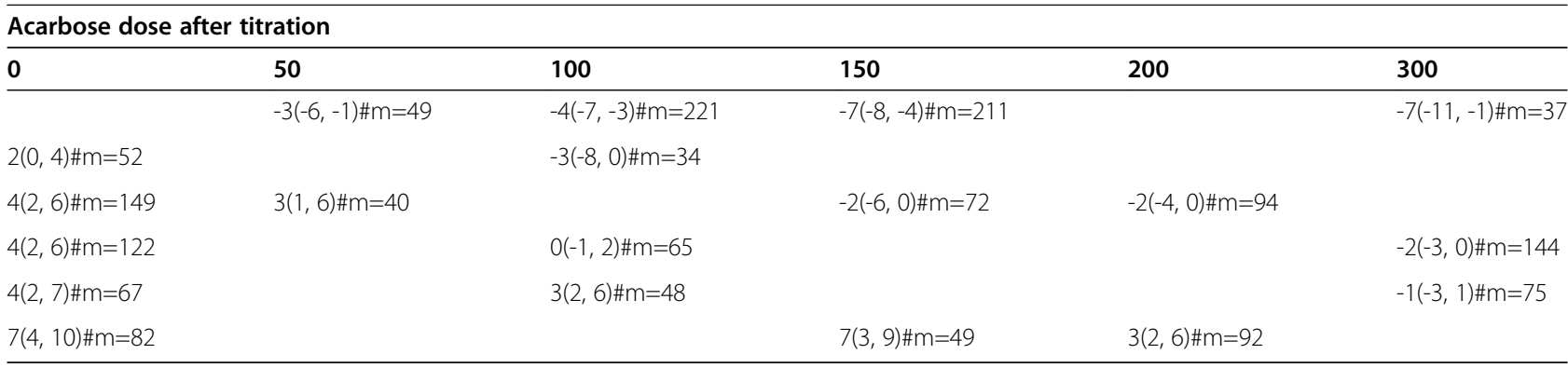

Change in $\mathrm{HbA1c}$ values after acarbose initiation, titration, or discontinuation. The values above the diagonal represent the instances where the medication has been initiated or up-titrated, while the values below the diagonal represent instances where the medication has been down-titrated or discontinued. The values refer to the mean difference in $\mathrm{HbA1C}(\mathrm{MD})$ and $95 \%$ confidence intervals. MD below 0 indicate a lowering in $\mathrm{HbA1c}$ while those above 0 indicate an increase in HbA1c. \#m refers to the number of HbA1c pairs for that dose titration

the only difference between the pre-titration and posttitration $\mathrm{HbA1c}$ was related to a single OAD dose titration.

Beyond clinical outcome focusing on glycemic control, resources required to deliberate $\mathrm{OAD}$ dose titration including additional time required in the shared decisionmaking process and the training of the physicians to competently address the concerns need to be examined in future research. The results from this study are collated and condensed for easy reference in Fig. 3. We envision that physicians would refer to this executive summary in clinical practice as they propose OAD initiation and titration to patients with the goal of achieving HbA1c targets. Compared to trial-based studies, the expected outcomes from real-world experience may be more relatable to everyday patients. Going further, this method of using real-world data to generate real-world evidence may be applied in other settings to costeffectively provide localized medication treatment effectiveness information. In the meantime, the investigators are currently developing an artificial intelligence-based counseling tool that will incorporate the study results to

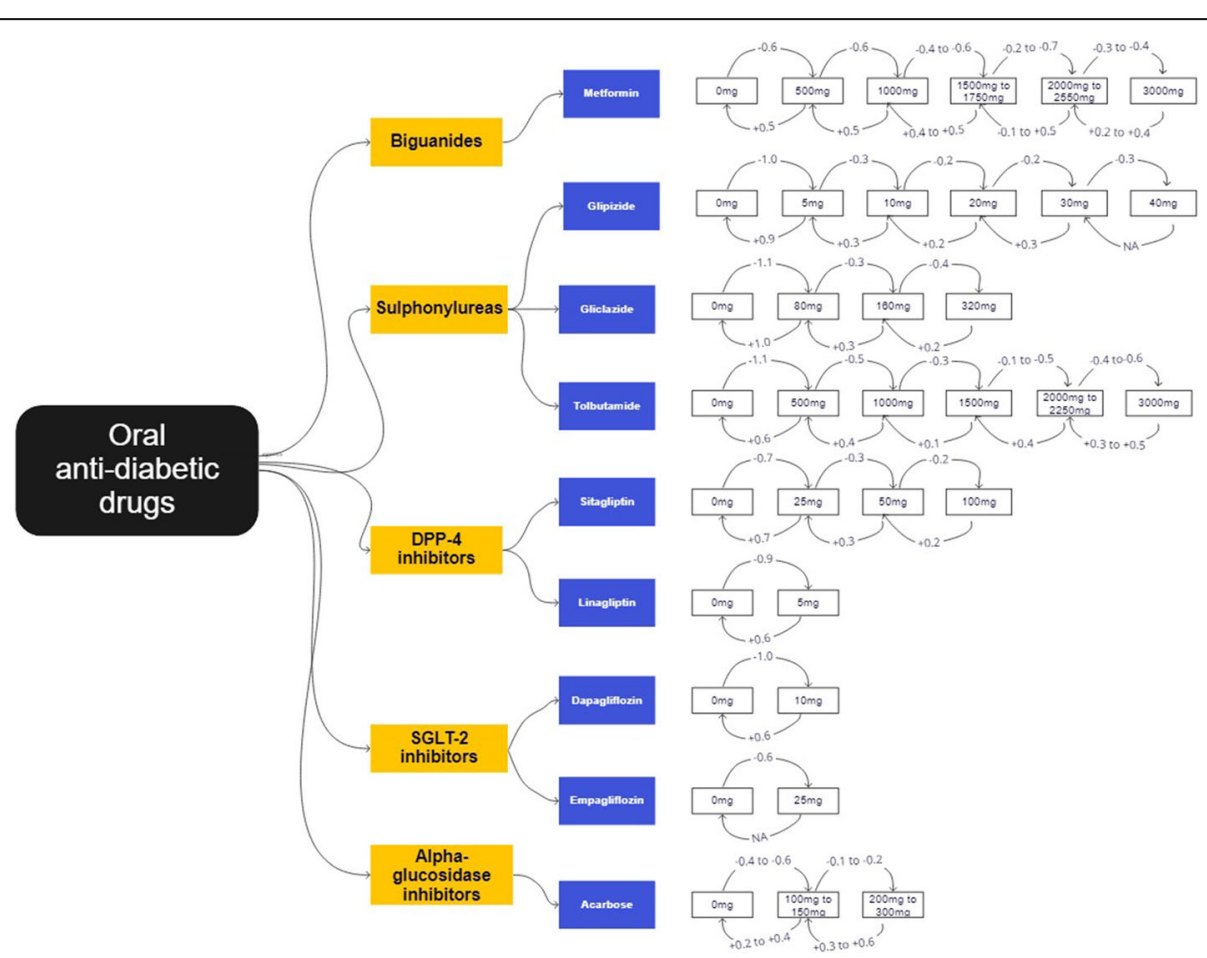

Fig. 3 Executive summary of $\mathrm{HbA} 1 \mathrm{c}$ change (with $\mathrm{HbA} 1 \mathrm{c}$ denoted in $\mathrm{mmol} / \mathrm{mol}$ ) with various $\mathrm{OAD}$ titration. The dosages in the white rectangle boxes refer to the total daily dosage of the medication. The numbers on the arrows represent the HbA1c change. The direction of the arrows represents an up-titration (rightward arrow), or down-titration (leftward arrow). Abbreviations: $\mathrm{HbA1c}=$ glycated haemoglobin, OAD = oral anti-diabetic drug 
better empower both patients and physicians with information to decide on their preferred OAD therapy, so as to achieve optimal clinical outcomes, minimal adverse effects, and best possible patient-centered care.

\section{Conclusions}

This study provides real-world evidence to elucidate the effect of OAD dose titration on HbA1c levels in Asian patients with T2DM. The real-world data in primary care showed lower HbA1c reduction after OAD dose escalation compared to results reported in clinical trials. In addition, this study also provides insights into OAD down-titration and discontinuation, which results in lower impact on HbA1c level than its corresponding uptitration or initiation. In clinical practice, these findings set realistic expectations for physicians to achieve the glycemic control of their patients during OAD dose titrations.

\section{Abbreviations}

DPP-4 inhibitor: Dipeptidyl peptidase-4 inhibitor; EMR: Electronic medical records; HbA1c: Glycated haemoglobin; ICD-10: International Classification of Diseases, $10^{\text {th }}$ Revision; NGSP: National Glycohemoglobin Standardization Program; NICE: National Institute for Health and Care Excellence; OAD: Oral anti-diabetic drug; SGLT-2 inhibitor: Sodium-glucose cotransporter-2 inhibitor; T2DM: Type-2 diabetes mellitus

\section{Supplementary Information}

The online version contains supplementary material available at https://doi. org/10.1186/s12916-021-02221-z.

Additional file 1. Additional file 1 of mean difference in $\mathrm{HbA1c}$ for the various $\mathrm{OAD}$ titrations and an executive summary denoted in $\%$ units. Figure S1 and Tables S1a to S1i. Figure S1. An executive summary of the results illustrating typical OAD titrations (with HbA1c denoted in \%). Tables S1a to S1i. Changes in HbA1c values (with HbA1c denoted in \%) after metformin titration.

Additional file 2. Additional file 2 of results from the analysis of "No OAD" and "Non-titrator" cohorts and multivariate analysis. Table S1, Figure S1, Table S2 and Table S3. Table S1. Change in HbA1c values (with $\mathrm{HbA1c}$ denoted in $\mathrm{mmol} / \mathrm{mol}$ ) in "No OAD" and "Non-titrators" cohorts. Figure S1. Visualization of covariates adjusted for in multivariate regression. Table S2. Multiple regression covariate weights (with HbA1c denoted in $\mathrm{mmol} / \mathrm{mol}$ ). Tables S3a to S3i. Change in $\mathrm{HbA1c}$ values (with $\mathrm{HbA} 1 \mathrm{c}$ denoted in $\mathrm{mmol} / \mathrm{mol}$ ) after metformin titration (adjusted by regression).

\section{Acknowledgements}

We would like to thank Ms. Patricia Kin, Ms. Caris Tan, Ms. Usha Sankari, and Mr. Aau Wai Keong from SingHealth Polyclinics Research Department for their support in making this work possible.

\section{Authors' contributions}

FHSA and TNC conceptualized and designed the study, with input from GQ, TWY, HW, and LML. GQ and TWY performed the data analysis. FHSA and GQ wrote the initial draft of the paper, to which the rest of the authors provided comments. All authors reviewed and approved the final manuscript.

\section{Funding}

This research is funded by the National Research Foundation, Singapore under its Al Singapore Programme (AISG Award No: AISG-GC-2019-001). Any opinions, findings, and conclusions or recommendations expressed in this material are those of the authors and do not reflect the views of National Research Foundation, Singapore.

\section{Availability of data and materials}

The datasets analyzed during the current study are not publicly available as they contain information that are sensitive to the study institution. They may be made available from the corresponding author on reasonable request.

\section{Declarations}

\section{Ethics approval and consent to participate}

Ethics approval was obtained from SingHealth Centralized Institution Review Board (CIRB) in 2019 (SingHealth CIRB Reference: 2019/2604). Patient consent was not obtained as the analysis was conducted on de-identified data.

\section{Consent for publication}

Not applicable.

\section{Competing interests}

The authors declare that they have no competing interests.

\section{Author details}

${ }^{1}$ SingHealth Polyclinics, SingHealth, 167, Jalan Bukit Merah, Connection One, Tower 5, \#15-10, Singapore P.O. 150167, Singapore. ${ }^{2}$ Institute of Data Science, National University of Singapore, Singapore, Singapore. ${ }^{3}$ School of Computing, National University of Singapore, Singapore, Singapore. ${ }^{4}$ Family Medicine Academic Clinical Programme, SingHealth-Duke NUS Academic Medical Centre, Singapore, Singapore.

Received: 12 July 2021 Accepted: 15 December 2021

Published online: 26 January 2022

\section{References}

1. Saeedi P, Petersohn I, Salpea P, Malanda B, Karuranga S, Unwin N, et al. Global and regional diabetes prevalence estimates for 2019 and projections for 2030 and 2045: results from the International Diabetes Federation Diabetes Atlas, 9th edition. Diabetes Res Clin Pract. 2019;157:107843.

2. Williams R, Karuranga S, Malanda B, Saeedi P, Basit A, Besançon S, et al. Global and regional estimates and projections of diabetes-related health expenditure: results from the International Diabetes Federation Diabetes Atlas, 9th edition. Diabetes Res Clin Pract. 2020;162:108072.

3. National Institute for Health and Care Excellence (UK). Type 2 diabetes in adults: management [Internet]. London: National Institute for Health and Care Excellence (UK); 2015 [cited 2021 May 21]. (National Institute for Health and Care Excellence: Clinical Guidelines). Available from: http://www.ncbi. nlm.nih.gov/books/NBK338142/

4. American Diabetes Association. 9. Pharmacologic approaches to glycemic treatment: standards of medical care in diabetes-2021. Diabetes Care. 2021: 44(Suppl 1):S111-24

5. Goh SY, Ang SB, Bee YM, Chen YT, Gardner DS, Ho ET, et al. Ministry of health clinical practice guidelines: diabetes mellitus. Singapore Med J. 2014; 55(6):334-47. https://doi.org/10.11622/smedj.2014079.

6. Sherifali D, Nerenberg K, Pullenayegum E, Cheng JE, Gerstein HC. The effect of oral antidiabetic agents on A1C levels: a systematic review and metaanalysis. Diabetes Care. 2010;33(8):1859-64. https://doi.org/10.2337/dc09-172 7.

7. Jia Y, Lao Y, Zhu H, Li N, Leung S-W. Is metformin still the most efficacious first-line oral hypoglycaemic drug in treating type 2 diabetes? A network meta-analysis of randomized controlled trials. Obes Rev Off J Int Assoc Study Obes. 2019 Jan;20(1):1-12. https://doi.org/10.1111/obr.12753.

8. Palanisamy S, Yien ELH, Shi LW, Si LY, Qi SH, Ling LSC, et al. Systematic review of efficacy and safety of newer antidiabetic drugs approved from 2013 to 2017 in controlling HbA1c in diabetes patients. Pharm Basel Switz. 2018;27(3):6(3). https://doi.org/10.3390/pharmacy6030057.

9. Ueshima H, Sekikawa A, Miura K, Turin TC, Takashima N, Kita Y, et al. Cardiovascular Disease and Risk Factors in Asia: A Selected Review. Circulation. 2008; 1 18(25):2702-9. https://doi.org/10.1161/CIRCULATIONAHA.1 08.790048.

10. Meadows TA, Bhatt DL, Cannon CP, Gersh BJ, Röther J, Goto S, et al. Ethnic differences in cardiovascular risks and mortality in atherothrombotic disease: insights from the REduction of Atherothrombosis for Continued Health 
(REACH) Registry. Mayo Clin Proc. 2011;86(10):960-7. https://doi.org/10.4065/ mcp.2011.0010.

11. Lee JWR, Brancati FL, Yeh H-C. Trends in the prevalence of type 2 diabetes in Asians versus Whites. Diabetes Care. 2011;34(2):353-7. https://doi.org/1 0.2337/dc10-0746.

12. Unnikrishnan R, Gupta PK, Mohan V. Diabetes in South Asians: phenotype, clinical presentation, and natural history. Curr Diab Rep. 2018;18(6):30. https://doi.org/10.1007/s1 1892-018-1002-8

13. Narayan KMV, Kanaya AM. Why are South Asians prone to type 2 diabetes? A hypothesis based on underexplored pathways. Diabetologia. 2020;63(6): 1103-9. https://doi.org/10.1007/s00125-020-05132-5.

14. Chandie Shaw PK, Baboe F, van Es LA, van der Vijver JC, van de Ree MA, de Jonge $\mathrm{N}$, et al. South-Asian type 2 diabetic patients have higher incidence and faster progression of renal disease compared with Dutch-European diabetic patients. Diabetes Care. 2006;29(6):1383-5. https://doi.org/10.2337/ dc06-0003.

15. Hu FB. Globalization of diabetes: the role of diet, lifestyle, and genes. Diabetes Care. 2011;34(6):1249-57. https://doi.org/10.2337/dc11-0442.

16. SingHealth Duke-NUS Academic Medical Centre Annual Report 2019/2020 Stronger Together. SingHealth. 2020. https://www.singhealth.com.sg/aboutsinghealth/newsroom/Documents/SingHealth\%20Duke-NUS\%20AR\%20201 9-20.pdf.

17. Bunn HF, Haney DN, Kamin S, Gabbay KH, Gallop PM. The biosynthesis of human hemoglobin A1c. Slow glycosylation of hemoglobin in vivo. J Clin Invest. 1976;57(6):1652-9. https://doi.org/10.1172/JCl108436.

18. Kaiafa G, Veneti S, Polychronopoulos G, Pilalas D, Daios S, Kanellos I, et al. Is $\mathrm{HbA1C}$ an ideal biomarker of well-controlled diabetes. Postgrad Med J. 2021;97(1148):380-3. https://doi.org/10.1136/postgradmedj-2020-138756.

19. Hirst JA, Farmer AJ, Ali R, Roberts NW, Stevens RJ. Quantifying the effect of metformin treatment and dose on glycemic control. Diabetes Care. 2012; 35(2):446-54. https://doi.org/10.2337/dc11-1465.

20. Hirst JA, Farmer AJ, Dyar A, Lung TWC, Stevens RJ. Estimating the effect of sulfonylurea on HbA1c in diabetes: a systematic review and meta-analysis. Diabetologia. 2013;56(5):973-84. https://doi.org/10.1007/s00125-013-2856-6.

21. Ji L, Ma J, Li H, Mansfield TA, T'joen CL, Iqbal N, et al. Dapagliflozin as monotherapy in drug-naive Asian patients with type 2 diabetes mellitus: a randomized, blinded, prospective phase III study. Clin Ther. 2014;36(1):84100 e9.

22. Lee CS, Tan JHM, Sankari U, Koh YLE, Tan NC. Assessing oral medication adherence among patients with type 2 diabetes mellitus treated with polytherapy in a developed Asian community: a cross-sectional study. BMJ Open. 2017;7(9):e016317. https://doi.org/10.1136/bmjopen-2017-016317.

23. Araki E, Onishi Y, Asano M, Kim H, Ekholm E, Johnsson E, et al. Efficacy and safety of dapagliflozin in addition to insulin therapy in Japanese patients with type 2 diabetes: results of the interim analysis of 16-week double-blind treatment period. J Diabetes Investig. 2016;7(4):555-64. https://doi.org/1 $0.1111 / j d i .12453$

24. Rosenstock J, Jelaska A, Zeller C, Kim G, Broedl UC, Woerle HJ, et al. Impact of empagliflozin added on to basal insulin in type 2 diabetes inadequately controlled on basal insulin: a 78-week randomized, double-blind, placebocontrolled trial. Diabetes Obes Metab. 2015;17(10):936-48. https://doi.org/1 $0.1111 /$ dom. 12503 .

25. Raz I, Hanefeld M, Xu L, Caria C, Williams-Herman D, Khatami H, et al. Efficacy and safety of the dipeptidyl peptidase-4 inhibitor sitagliptin as monotherapy in patients with type 2 diabetes mellitus. Diabetologia. 2006; 49(11):2564-71. https://doi.org/10.1007/s00125-006-0416-z.

26. García-Pérez L-E, Alvarez M, Dilla T, Gil-Guillén V, Orozco-Beltrán D. Adherence to therapies in patients with type 2 diabetes. Diabetes Ther Res Treat Educ Diabetes Relat Disord. 2013;4(2):175-94. https://doi.org/10.1007/ s13300-013-0034-y.

27. Kim K, Unni S, Brixner DI, Thomas SM, Olsen CJ, Sterling KL, et al. Longitudinal changes in glycated haemoglobin following treatment intensification after inadequate response to two oral antidiabetic agents in patients with type 2 diabetes. Diabetes Obes Metab. 2019;21(7):1725-33. https://doi.org/10.1111/dom.13694.

28. Kim K, Johnson JA, Derendorf H. Differences in drug pharmacokinetics between East Asians and Caucasians and the role of genetic polymorphisms. J Clin Pharmacol. 2004;44(10):1083-105. https://doi.org/1 $0.1177 / 0091270004268128$.

29. Sarashina A, Friedrich C, Crowe S, Patel S, Graefe-Mody U, Hayashi N, et al. Comparable pharmacodynamics, efficacy, and safety of linagliptin $5 \mathrm{mg}$ among Japanese, Asian and white patients with type 2 diabetes. J Diabetes Investig. 2016;7(5):744-50. https://doi.org/10.1111/jdi.12482.

30. Mostafavi F, Alavijeh FZ, Salahshouri A, Mahaki B. The psychosocial barriers to medication adherence of patients with type 2 diabetes: a qualitative study. Biopsychosoc Med. 2021;15(1):1. https://doi.org/10.1186/s13030-02000202-X.

31. Blonde L, Khunti K, Harris SB, Meizinger C, Skolnik NS. Interpretation and impact of real-world clinical data for the practicing clinician. Adv Ther. 2018; 35(11):1763-74. https://doi.org/10.1007/s12325-018-0805-y.

32. Ong CR, Molyneaux LM, Constantino MI, Twigg SM, Yue DK. Long-term efficacy of metformin therapy in nonobese individuals with type 2 diabetes. Diabetes Care. 2006;29(11):2361-4. https://doi.org/10.2337/dc06-0827.

33. Ito H, Ishida $\mathrm{H}$, Takeuchi Y, Antoku S, Abe M, Mifune M, et al. Long-term effect of metformin on blood glucose control in non-obese patients with type 2 diabetes mellitus. Nutr Metab. 2010;7(1):83. https://doi.org/10.1186/1 743-7075-7-83.

34. Desai U, Kirson NY, Kim J, Khunti K, King S, Trieschman E, et al. Time to treatment intensification after monotherapy failure and its association with subsequent glycemic control among 93,515 patients with type 2 diabetes. Diabetes Care. 2018;41(10):2096-104. https://doi.org/10.2337/dc17-0662.

35. Ferrannini E, Berk A, Hantel S, Pinnetti S, Hach T, Woerle HJ, et al. Long-term safety and efficacy of empagliflozin, sitagliptin, and metformin: an activecontrolled, parallel-group, randomized, 78-week open-label extension study in patients with type 2 diabetes. Diabetes Care. 2013;36(12):4015-21. https:// doi.org/10.2337/dc13-0663

\section{Publisher's Note}

Springer Nature remains neutral with regard to jurisdictional claims in published maps and institutional affiliations.

\section{Ready to submit your research? Choose BMC and benefit from:}

- fast, convenient online submission

- thorough peer review by experienced researchers in your field

- rapid publication on acceptance

- support for research data, including large and complex data types

- gold Open Access which fosters wider collaboration and increased citations

- maximum visibility for your research: over $100 \mathrm{M}$ website views per year

At $\mathrm{BMC}$, research is always in progress.

Learn more biomedcentral.com/submissions 